\title{
Phenanthrenequinone-Based Linear Polymers as Sustainable Cathode Materials for Rechargeable Li-Ion Batteries
}

\author{
Xinya Guo ${ }^{1}$, Ying Zhang ${ }^{1}$, Han Chen ${ }^{1}$, Chaohui Cui ${ }^{2}$, Zhenyao $\mathrm{Li}^{1}, \mathrm{Ya} \mathrm{Du}^{2, *}$, \\ Baofeng Wang ${ }^{1, *}$ and Haishen Yang ${ }^{1, *}$ \\ ${ }^{1}$ Shanghai Key Laboratory of Materials Protection and Advanced Materials in Electric Power, College \\ of Environmental and Chemical Engineering, Shanghai University of Electric Power, Shanghai \\ 200090, China. \\ ${ }^{2}$ Institute of Advanced Synthesis, School of Chemistry and Molecular Engineering, Nanjing Tech \\ University, Nanjing 211816, China. \\ *E-mail: yanghsh@shiep.edu.cn (H. Yang), wangbaofeng@ shiep.edu.cn (B. Wang), \\ ias_ydu@njtech.edu.cn (Y.Du)
}

doi: $10.20964 / 2020.08 .75$

Received: 17 April 2020 / Accepted: 2 June 2020 / Published: 10 July 2020

\begin{abstract}
Coal tar is a useful industrial by-product, which is produced in large quantities every year, but has not been adequately utilized thus far. Herein, we design and synthesize two novel coaltar-derived organic linear polymers, namely, poly(2-ethynylphenanthrene-9,10-dione) (PEPQ) and poly(2-(thiophen-2yl)phenanthrene-9,10-dione) (PTPQ). Beyond as traditional anode materials from coal tar, PEPQ and PTPQ were studied as lithium (Li)-ion battery (LIB) cathode materials in this study. The obtained PEPQ and PTPQ exhibited reversible specific capacities of up to 125 and $138 \mathrm{mAh} \mathrm{g}^{-1}$, respectively, at $10 \mathrm{~mA} \mathrm{~g}^{-1}$, in LIBs. In particular, the comparative study on the electrochemical performance of these two polymers revealed that replacing the ethynylene linkage (in PEPQ) with thiophene linkage (in PTPQ) endowed the material with faster redox kinetics, a greater electronic conductivity, and thus a higher active site utilization ( $75 \%$ vs. $54 \%$ ). This discovery in this work could provide elementary insights into the rational construction of organic cathode materials for LIBs with a superior performance.
\end{abstract}

Keywords: Coal Tar, Linear Conjugated Polymer, Lithium Ion Batteries, Organic Cathode Material.

\section{$\underline{\text { FULL TEXT }}$}

(C) 2020 The Authors. Published by ESG (www.electrochemsci.org). This article is an open access article distributed under the terms and conditions of the Creative Commons Attribution license (http://creativecommons.org/licenses/by/4.0/). 